\title{
Trophic-specific responses to migration in empirical metacommunities
}

Zachary Hajian-Forooshani ${ }^{1}$, Lauren Schmitt ${ }^{2}$, Nicholas Medina ${ }^{1}$ and John Vandermeer ${ }^{1}$

${ }^{1}$ Dept of Ecology and Evolutionary Biology, Univ. of Michigan, 1105 N University Ave., Ann Arbor, MI 48109, USA

${ }^{2}$ School for Environment and Sustainability, Univ. of Michigan, Ann Arbor, MI, USA

Corresponding author: Zachary Hajian-Forooshani, Dept of Ecology and Evolutionary Biology, Univ. of Michigan, 1105 N University Ave., Ann Arbor, MI 48109, USA. E-mail: zhajianf@umich.edu

Decision date: $22-\mathrm{Nov}-2019$

This is the author manuscript accepted for publication and has undergone full peer review but has not been through the copyediting, typesetting, pagination and proofreading process, which may lead to differences between this version and the Version of Record. Please cite this article as doi: [10.1111/oik.06342].

'This article is protected by copyright. All rights reserved.' 


\section{Abstract}

The metacommunity, as it evolved from Levins's metapopulation, provides a framework to consider the spatial organization of species interactions. A defining feature of metapopulations and metacommunities is that organisms (populations or communities) are connected via migration. An important result from Levins's metapopulation work - that increasing migration lowers regional extinction probability - is often incorporated into conceptions of metacommunities; however, this may not hold true for multiple interacting metapopulations (metacommunities). We report results from a metacommunity field experiment conducted with a tropical terrestrial leaf litter macro-arthropod community. We show that migration induces regional extinctions of predators without changing the predator community composition. For non-predators we found no evidence of regional extinctions, but a significant change in community composition. Our result corroborates the findings of a prior similar metacommunity experiment with a temperate forest leaf litter community. The concordance between these experiments, even with vastly different communities, highlights the importance of considering trophic and non-trophic community structure to understand metacommunity dynamics, and suggests a potential connection between migration rates and trophicspecific responses in ecological communities.

Keywords: metacommunity, metapopulation, migration, non-trophic interactions, space 


\section{Introduction}

The theory of metapopulations has become a standard way of thinking about simple population dynamics, and its success has stimulated a seemingly obvious extension, the metacommunity (Levins 1969; Wilson 1992). As originally envisioned, a metacommunity is a collection of interacting populations of different species in which extinction and migration occur on a regular basis. This results in patchiness that creates subcommunities, which may be distinct in species composition. It might be argued that MacArthur and Wilson's theory of island biogeography was the first metacommunity theory, and perhaps the most elegant, in which patchiness is provided by the existence of islands (MacArthur and Wilson 1964). From these formulations, an important conclusion of metapopulation theory has been tacitly incorporated as an obvious corollary of metacommunity theory - that increasing migration lowers overall extinction probability. While it may be a reasonable proposition at first glance, further reflection on the assumption suggests that the expectation of lowered extinction is not universal in the metacommunity context (Vandermeer et al. 1980; Caswell and Cohen 1991).

Huffaker's classic experiment might be thought of as a canonical case study that supports a link between increasing inter-patch migration and lower extinction. When isolated to a small feeding area, both predator and prey mites go extinct (predator eats prey to extinction then itself goes locally extinct)-yet when many smaller feeding areas were arranged to facilitate local dispersal of prey (but not predators), apparently stable oscillations result (Huffaker 1958). A similar experimental set-up that yielded stable oscillations also included predator-focused dispersal limitations (Huffaker 1958). A similar pattern was also observed in Gause's experimental system of protozoans twenty years prior (Gause et al. 1936). In these examples, migration appears to decrease the probability of extinction.

Alternatively, it is not difficult to imagine the reverse outcome in other systems: a case in which migration might increase extinction probability. For example, in a two-predator one-prey situation in which spatial structure allows for a segregation of the two predators in space, increasing the predator migration rate could increase intraguild antagonism, leading to one of the predators dominating, and a reduction of total species diversity from three to two. Even in cases without intraguild competition, the simple dispersal of any intermediate predator can theoretically cause trophic instabilities due to more lag in population dynamics (Jansen 1995). Thus, elevated migration rates could result in either increased or decreased species diversity, depending on the strength or timescale of antagonistic (or even facilitative) interactions across ecological guilds (Guzman et al. 2019). The simple migration-extinction equilibrium of island biogeography and metapopulation theory may yield predictions that are not generalizable for more complex community structures.

The role of migration in rescuing unstable populations has been highlighted so often that it is usually taken for granted, but that result is not theoretically inevitable (Simberloff and Cox 1987). The ability to simulate a wide range of metacommunity dynamics suggests the need for an empirical approach. One of the key shortfalls of much of the experimental metacommunity work lies in its simplification of community interactions (Polis et al. 1989), where studies often only consider a subset of species with simple trophic structure (e.g. consumer-resource pairs) Warren 1996; Shurin 2001; Kneitel and Miller 2003; Cadotte 2006; Fox et al. 2017). Although there are some notable experiments which attempt to include some of the trophic and non-trophic realism of communities (Neill 1974; Vandermeer et al. 1980), surprisingly few experimental studies have focused on empirically realistic metacommunities.

One of the early attempts to study the role of migration in empirical metacommunities used leaf litter macro-arthropod communities and found that the predator guild (defined taxonomically) decreased in richness when random migration was induced, while non-predator richness was unaffected by migration (Vandermeer et al. 1980). These results suggest that conclusions about metacommunity structure may, at least in some contexts, be trophic-specific. The dependence of community dynamics on trophic structure has been noted in some well-known debates, for example, in considering whether communities tend to be controlled primarily by consumers (top-down) or producers (bottom-up) (Hairston et al. 1960), and why trophic cascades operate differently in aquatic versus terrestrial systems (Strong 1992). Clearly, a wide range of ecological processes interact with migration in real metacommunities, including higher-order interactions (or trait-mediated indirect interactions), which recent work suggests may be more determinant of community structure than the more direct, lower-order or pairwise species interactions (Werner and Peacor 2003; Bairey et al. 2016; Grilli et al. 2017; Terry et al. 2017).

The empirical result that experimental migrations make a difference in metacommunity structure for predators, but not for non-predators (Vandermeer et al. 1980), was found in a species-poor temperate deciduous forest (Michigan, USA). Here we revisit the experiment conducted by Vandermeer et al. (1980) in a more speciose montane tropical agroecosystem. We considered that in the tropics, the hypothesized stability-preserving aspect of high biodiversity (McCann 2000) could overwhelm any special effect of a strongly antagonistic predator species, which was proposed to explain the results of the earlier temperate zone study. Accordingly, we sought to investigate how migration affects leaf litter community richness and composition, focusing especially on the effects at different trophic levels. Based 
on underlying assumptions about the importance of migration in maintaining biodiversity, we hypothesized that curtailing local migration would reduce local species diversity, and that this effect would be observed at all trophic levels.

\section{Methods}

Study region and design

This study was conducted at Finca Irlanda, an organic shaded coffee agroecosystem in the Soconusco region of Chiapas, Mexico. The study site was on a subset of land recently transitioned from rustic coffee production to a forested reserve. The experimental set-up was positioned adjacent to a patch of invasive golden bamboo, the litter of which created a uniform mat.

Leaf litter was collected from a well-forested area of the reserve, homogenized, and separated into 10 mesocosms, each of which were $0.5 \mathrm{~m}^{2}$ in area and separated from one another by 1 meter. Five mesocosms were positioned on either side of a walking trail. No physical barriers prevented migration between mesocosms. Inter-patch migration was therefore possible, but we assumed it was sufficiently infrequent such that it could be ignored. We assumed organisms would not prefer to leave a mesocosm of leaf litter of the same composition from which they were originally collected to migrate across a relatively inhospitable mat of dried litter from an invasive bamboo species.

Half of the mesocosms were assigned as controls and half as treatments. To simulate migration among treatments, one-quarter of the litter in each mesocosm was removed and replaced with the same amount of litter from a different mesocosm. The transfer schedule was set so that a different quarter of the mesocosm was migrated during each transfer event, and the replacement pattern was randomized so that each mesocosm received and contributed litter to a different, randomly assigned mesocosm. Transfers were done every 4 days for 16 days. Migration was not manipulated among control mesocosms, though one-quarter of the litter was lifted, agitated, and replaced in the same mesocosm every four days to control for the disturbance of the litter transfers between treatment mesocosms. All mesocosms were harvested on day 20. This time scale is comparable to Vandermeer et al.'s 1980 experiment which ran for 30 days.

After harvesting, the litter was sieved using 3-mm meshes to remove coarse detritus. Each sample was searched by four people for 20 minutes, and all encountered organisms were individually removed and placed in alcohol. This technique did not likely capture all organisms found within the mesocosms, but we expect that any bias toward certain groups of organisms was standardized across all samples, which we assured by blinding the sample labels throughout the sorting process. Individuals were sorted into orders or families and identified to morphospecies. Morphospecies were then classified as either predators or non-predators, where predators included spiders, Staphylinidae beetle larvae, pseudoscorpions and centipedes.

\section{Statistical methods}

To compare the number of species in our control and migration treatments, individual-based rarefaction curves were calculated for the whole dataset and for each trophic group (predators and nonpredators) separately. Rarefactions followed the now standard methodology of resampling the list of species observations with replacement at increasing numbers of individuals (Gotelli and Colwell 2001). 100 resamples were conducted for each level of individuals sampled, and the mean number of species for a given density of individuals was calculated.

While there are standard methods to extrapolate the number of species (Colwell and Coddington 1994; Chao et al. 2014) and compare the overall shape of rarefaction curves (Cayuela et al. 2015), we were interested in the statistical differences between our rarefaction curves across the range of resampled levels. To assess differences between the control and migration treatments, we conducted a bootstrapping procedure to compare the difference in the mean number of species at every re-sampling level, $x_{i}$, along the rarefaction curves. For a given $x_{i}, 100$ random draws from the observed datasets for the control and migration, $D_{c}$ and $D_{m}$, were used to calculate the mean observed number of species, $\overline{S_{c}}$ and $\overline{S_{m}}$, for sampling level $x_{i}$. These values were used to calculate the observed difference in the number of species, $\overline{S_{c}}-\overline{S_{m}}=\Delta_{O B S}$ for resampling level $x_{i}$. The observed data, $D_{c}$ and $D_{m}$, were then pooled together to create $D_{p}$, which was then randomly partitioned into null data sets 100 times for the control and migration treatments, $N_{c}$ and $N_{m} . N_{c}$ and $N_{m}$ were then randomly sampled 100 times at $x_{i}$ sampling level to calculate mean number of species, $\overline{S_{N_{c}}}$ and $\overline{S_{N_{m}}}$, sampled at $x_{i}$ for both null data sets. These values were then used to calculate the null difference in the mean number of species sampled, $\overline{S_{N_{c}}}-\overline{S_{N_{m}}}=\Delta_{N U L L}$. This gives us a distribution of $\Delta_{N U L L}$ which was then compared to $\Delta_{O B S}$ to calculate the probability of observing $\Delta_{O B S}$ for a given $x_{i}$ if $D_{c}$ and $D_{m}$ come from the same statistical population. The probability, $p$, is calculated by $p=\frac{1}{n} \sum q$ where $q$ is the number of times that $\Delta_{O B S} \geq \Delta_{N U L L}$ and $n$ is the number of $\Delta_{N U L L}$ values in the distribution. This procedure was repeated for every value of $x_{i}$ where the rarefactions of both treatments overlap. The supplementary material contains a graphical walkthrough of the statistical test (Figure S1) and a link to a repository with the R code for the test. 
dynamics. The type of detailed observations of community interactions that would be necessary to understand the mechanisms that generated our experimental results were not feasible in our study; we could only look at patterns of community richness and composition to attempt to shed light on potential mechanisms at play. The two key findings are that first, predator richness decreased significantly with migration, but community structure did not significantly change, and secondly, while non-predator richness did not change significantly, community structure did (Figure $1 \&$ Figure 2). For the predator community, this suggests that although there is a reduction in the number of morpho-species, the relative abundance and presence in the rest of the community was not significantly impacted by migration. This indicates that migration may have had a very species-specific impact within the predator guild, the effects of which then rippled through the non-predator community. We suggest that a highly antagonistic and relatively rare (possibly initially isolated to only a single patch) predator may be shaping the community when dispersed among patches, as was suggested in Vandermeer et al. (1980). Support for this hypothesis is in the higher percentage of rare predator morpho-species in the treatment with no migration (Table 1.). Non-significant compositional changes in the predator community may be the result of the extinction of relatively rare predator morpho-species, an effect not present in the non-predator community. While rare predators are impacted by migration, rare non-predators are not. The $\mathrm{R}$ statistics reported from the ANOSIM tests suggest a similar story, where the larger R for the Jaccard index compared to Bray-Curtis observed in the predator community suggest targeted species specific changes to the communities.

Based on patterns in community richness and composition, we suggest that an antagonist and relatively rare predator may be shaping these leaf litter communities, but it remains that both trophic and non-trophic mechanisms acting within and between guilds could be drawn upon to explain these results. Intraguild effects among predator communities are common and may manifest in the form of indirect competition among predators or intraguild predation. Impacts of predators on community structure are often hypothesized as acting through lower trophic levels such as predator-mediated coexistence or keystone predation (Shurin and Allen 2002). It is also possible that the changes observed resulted from non-trophic interactions such as trait-mediated indirect interactions (Werner and Peacor 2003; Bairey et al. 2016; Grilli et al. 2017; Terry et al. 2017), which may be acting within or between trophic levels. As is widely documented in ecological communities, the addition of new species (i.e. by migration) can result in local changes to the magnitude, and even sign, of other species' pairwise direct interactions, often caused by behavioral changes. For example, the mere presence of a predator at a low density can decrease the maximum observed foraging activity and thus fitness of a prey (Werner and Peacor 2003). In a metacommunity context, higher-order anti-predator effects have also been shown to shape metacommunity dynamics when migration is induced in simple experimental systems (Kneitel and Miller 2003; Hauzy et al. 2007; Howeth and Leibold 2010). If there are differences in the structure of indirect interactions within trophic levels, we may predict different dynamics for each trophic level. For instance, there may be more strong negative indirect interactions among predators in a system, but weaker positive indirect effects among prey; this could cause more exclusion and lower richness among predators, yet little to no change in prey richness. Ultimately, a myriad of trophic and non-trophic mechanisms may be important in shaping metacommunity dynamics, and we emphasize their consideration in developing modern metacommunity theory (Guzman et al. 2019) .

Community organization, which encompasses the ways in which trophic levels are connected across scales also mediates the ways in which predators shape metacommunity dynamics (Shurin 2001, Shurin and Allen 2001, CadotteCodetta and Fukami 2005). The context dependency associated with metacommunity dynamics is likely a reflection of the complex ways in which communities can be organized trophically and non-trophically. The ability to account for the true distribution of interactions in ecosystems is likely limited in natural systems, but will further our understanding of the plethora of theoretical and empirical results regarding trophically specific roles in metacommunities (Shurin 2001; Kneitel and Miller; Caswell 1978; Caswell and Cohen 1991). Most frequently, experimentalists look at the impacts of predators on the overall diversity of the metacommunity, and have found examples of predators increasing regional diversity (Shurin 2001) as well as decreasing it (Codette and Fukami 2005). What has been less frequently explored is the impact of migration on the predator and non-predator guilds separately. Most hypotheses associated with trophic guilds and metacommunities are related to the rate of migration in the system or simply the presence of predators (Kneitel and Miller 2003). Regarding our study system, the relatively small literature on the community ecology of terrestrial leaf litter arthropod communities makes it difficult to narrow potential mechanisms that may explain our results.

In our experiment, we manipulated migration rates such that there were equivalent potential migration rates for predators and non-predators. This could be an important caveat to our study's generalizability, given the potential differences in the realized predator and non-predator migration rates. Migration should increase diversity initially, as homogenization occurs, but trophic and non-trophic interactions can act to increase or decrease diversity after this initial homogenization. Our result of lower predator richness with migration runs counter to this expectation of increased diversity with homogenization, and thus indicates that our experimental time frame was appropriate to assess changes in these communities. We found no change in the richness of non-predators, but have no reason to suspect 
that homogenization effects would operate on a different time scale for predators and non-predators in the leaf litter community, particularly given that we manipulated the potential migration rates to be equal across trophic levels.

The work reported herein sits comfortably with the current enthusiasm for metacommunities, a framework originally suggested by Wilson (1992). It is substantially similar to the framework of MacArthur and Wilson's original offering, in which 1) ecological dynamics occur locally, with species interactions (of various forms) determining which species will survive and which will locally perish, while 2) the more regional process of migration continually feeds these local communities, countering local extinctions with regional migrations to provide the expected equilibrium (MacArthur and Wilson 1964). Eschewing some recent complexities (Leibold et al. 2004), we consider a metacommunity as structured in the original sense of Wilson (1992), wherein ecological dynamics occur at a local level, but local patches affect one another through dispersal. Our experiment interrogates the consequences of migration, but more specifically explores the interaction between community structure and the dynamics of migration. Our results highlight the importance of considering trophic and non-trophic structure when evaluating metacommunity dynamics.

\section{Acknowledgments}

The authors with like to acknowledge: Don Jorgé for providing technical and logistical support in the development, construction and implementation of our experimental mesocosms, Marleni (Flor) Velasquez Soto for providing culinary support on the farm, Walter Peters of Finca Irlanda for allowing us to conduct this work on his farm, two anonymous reviewers who helped improve the manuscript, and Ferdinand LaMothe for providing creative support throughout the duration of the project.

\section{References}

Bairey, E., E. D. Kelsic, and R. Kishony. 2016. High-order species interactions shape ecosystem diversity. Nature Communications 7:12285.

Bowler, D. E., \& Benton, T. G. (2005). Causes and consequences of animal dispersal strategies: relating individual behaviour to spatial dynamics. Biological Reviews, 80(2), 205-225.

Cadotte, M. W. 2006. Metacommunity influences on community richness at multiple spatial scales: a microcosm experiment. Ecology 87(4):1008-1016.

Cadotte, M. W., \& Fukami, T. 2005. Dispersal, spatial scale, and species diversity in a hierarchically structured experimental landscape. Ecology Letters, 8(5): 548-557.

Caswell, H. and J.E. Cohen. 1991. Disturbance, interspecific interaction and diversity in metapopulations. In Metapopulation dynamics: Empirical and theoretical investigations (pp. 193-218).

Cayuela, L., N. J. Gotelli, and R.K. Colwell. 2015. Ecological and biogeographic null hypotheses for comparing rarefaction curves. Ecological Monographs 85(3): 437-455.

Colwell, R. K., and J.A. Coddington. 1994. Estimating terrestrial biodiversity through extrapolation. Phil. Trans. R. Soc. Lond. B 345(1311):101-118.

Chao, A., N.J. Gotelli, T.C. Hsieh, E. L. Sander, K.H. Ma, R. K. Colwell, and A.M. Ellison. 2014. Rarefaction and extrapolation with Hill numbers: a framework for sampling and estimation in species diversity studies. Ecological Monographs 84(1): 45-67.

Clobert, J., Le Galliard, J. F., Cote, J., Meylan, S., \& Massot, M. 2009. Informed dispersal, heterogeneity in animal dispersal syndromes and the dynamics of spatially structured populations. Ecology letters, 12(3):197-209.

Economo, E. P., and T.H. Keitt. 2008. Species diversity in neutral metacommunities: a network approach. Ecology letters 11(1):52-62.

Fox, J. W., D. Vasseur, M. Cotroneo, L. Guan, and F. Simon. 2017. Population extinctions can increase metapopulation persistence. Nature ecology \& evolution 1(9):1271.

Gause, G. F., N. P. Smaragdova, and A. A. Witt, A. A. 1936. Further studies of interaction between predators and prey. The Journal of Animal Ecology 1-18. 
Grilli, J., G. Barabás, M.J. Michalska-Smith, and S. Allesina. 2017. Higher-order interactions stabilize dynamics in competitive network models. Nature 548(7666):210.

Gotelli, N. J., and R.K. Colwell. 2001. Quantifying biodiversity: procedures and pitfalls in the measurement and comparison of species richness. Ecology letters 4(4):379-391.

Gouhier, T. C., F. Guichard, and A. Gonzalez. 2010. Synchrony and stability of food webs in metacommunities. The American Naturalist, 175(2): E16-E34.

Guzman, L. M., Germain, R. M., Forbes, C., Straus, S., O'Connor, M. I., Gravel, D., ... \& Thompson, P. L. 2019. Towards a multi-trophic extension of metacommunity ecology. Ecology letters, 22(1):19-33.

Hairston, N. G., F.E. Smith, and L.B. Slobodkin. 1960. Community structure, population control, and competition. The American Naturalist 94(879):421-425.

Hauzy, C., Hulot, F. D., Gins, A., \& Loreau, M. 2007. Intra-and interspecific density-dependent dispersal in an aquatic prey-predator system. Journal of Animal Ecology, 76(3):552-558.

Heatwole, H., and R. Levins. 1973. Biogeography of the Puerto Rican Bank: species-turnover on a small cay, Cayo Ahogado. Ecology 54(5):1042-1055.

Heatwole, H., and R. Levins.. 1972. Trophic structure stability and faunal change during recolonization. Ecology 53(3): 531-534.

Howeth, J. G., and M.A. Leibold. 2010. Prey dispersal rate affects prey species composition and trait diversity in response to multiple predators in metacommunities. Journal of Animal Ecology 79(5):10001011 .

Huffaker, C. 1958. Experimental studies on predation: dispersion factors and predator-prey oscillations. Hilgardia 27(14):343-383.

Kneitel, J. M., and T.E.Miller. 2003. Dispersal rates affect species composition in metacommunities of Sarracenia purpurea inquilines. The American Naturalist 162(2): 165-171.

Koelle, K., and J. Vandermeer. 2005. Dispersal-induced desynchronization: from metapopulations to metacommunities. Ecology Letters, 8(2): 167-175.

Leibold, M. A., M. Holyoak, N. Mouquet, P. Amarasekare, J.M. Chase, M.F. Hoopes, ... \& M. Loreau. 2004. The metacommunity concept: a framework for multi-scale community ecology. Ecology Letters 7(7):601-613.

Levins, R. 1969. Some demographic and genetic consequences of environmental heterogeneity for biological control. American Entomologist 15(3):237-240.

Levins, R., M.L. Pressick, and H. Heatwole. 1973. Coexistence Patterns in Insular Ants: In which it is shown that ants travel a lot from island to island, and what they do when they get there. American Scientist 61(4):463-472.

Levins, R., and H. Heatwole. 1973. Biogeography of the Puerto Rican bank: introduction of species onto Palominitos Island. Ecology 54(5):1056-1064.

MacArthur, R. H., and E.O. Wilson. 1964. The Theory of Island Biogeography (Vol. 1). Princeton University Press.

McCann, K. S. "The diversity-stability debate." Nature 405, no. 6783 (2000): 228.

Mouquet, N., and M. Loreau. 2002. Coexistence in metacommunities: the regional similarity hypothesis. The American Naturalist 159(4): 420-426.

Neill, W. E. 1974. The community matrix and interdependence of the competition coefficients. The American Naturalist 108(962): 399-408. 
Oksanen, Jari, F. Guillaume Blanchet, Roeland Kindt, Pierre Legendre, R. B. O’hara, Gavin L. Simpson, Peter Solymos, M. Henry H. Stevens, and Helene Wagner. "Vegan: community ecology package. R package version 1.17-4." http://cran. r-project. org>. Acesso em 23 (2010): 2010.

Polis, G. A., C.A. Myers, and R. D. Holt. 1989. The ecology and evolution of intraguild predation: potential competitors that eat each other. Annual review of ecology and systematics 20(1): 297-330.

Shurin, J. B. 2001. Interactive effects of predation and dispersal on zooplankton communities. Ecology 82(12):3404-3416.

Shurin, J. B., \& Allen, E. G. 2001. Effects of competition, predation, and dispersal on species richness at local and regional scales. The American Naturalist, 158(6): 624-637.

Simberloff, D., and J. Cox. 1987. Consequences and costs of conservation corridors. Conservation biology 1(1):63-71.

Steiner, C. F., R.D. Stockwell, V. Kalaimani, and Z. Aqel. 2013. Population synchrony and stability in environmentally forced metacommunities. Oikos, 122(8):1195-1206.

Strong, D. R. 1992. Are trophic cascades all wet? Differentiation and donor-control in speciose ecosystems. Ecology 73(3):747-754.

Terry, J. C. D., R.J. Morris, and M.B. Bonsall. 2017. Trophic interaction modifications: an empirical and theoretical framework. Ecology Letters 20(10):1219-1230.

Vandermeer, J., J. Lazarus, C. Ludwig, J. Lyon, B. Schultz, and K. Yih. 1980. Migration as a factor in the community structure of a macroarthropod litter fauna. The American Naturalist 115(4):606-612.

Warren, P. H. 1996. The effects of between-habitat dispersal rate on protist communities and metacommunities in microcosms at two spatial scales. Oecologia 105(1):132-140.

Werner, E. E., and S.D. Peacor. 2003. A review of trait-mediated indirect interactions in ecological communities. Ecology 84(5):1083-1100.

Wilson, D. S. 1992. Complex interactions in metacommunities, with implications for biodiversity and higher levels of selection. Ecology 73(6):1984-2000.

Supplementary material (available online as Appendix oik-06342 at <www.oikosjournal.org/appendix/oik-06342>). Appendix 1 


\section{Figure Legends}

Figure 1: (A-C) Individual-based rarefaction curves for A.) whole community (green), B.) predators (red), and C.) non-predators (blue). Treatments are shown in lighter colors (control) and darker colors (migration). One standard deviation (based on the 1000 random draws) is plotted in the shaded areas around the curves. The vertical dashes above the curves in in B.) represent a statistically significant ( $\mathrm{p}<$ 0.05 ) difference in the number of species for a given number of individuals sampled between the control and migration treatments.
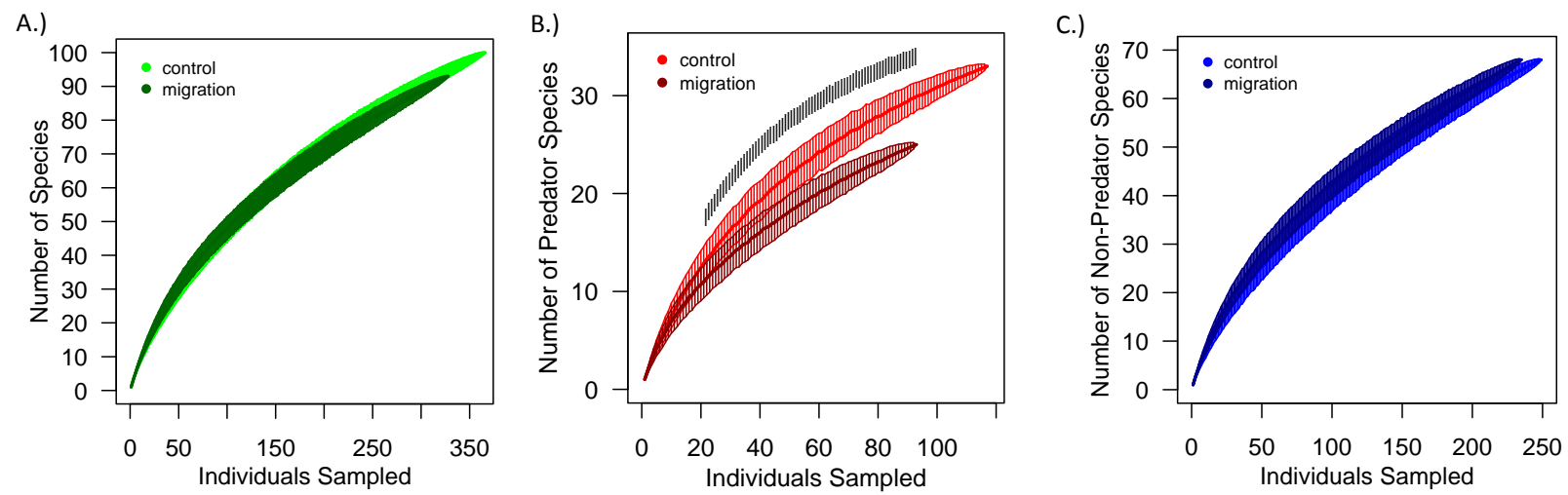
Figure 2: NMDS plots for predator community and non-predator community. a.) and b.) are made using Bray-Curtis dissimilarity and c.) and d.) are using Jaccard. ANOSIM showed no differences between control and migration treatments for the predators (Bray-Curtis $\mathrm{R}=0.12 ; \mathrm{p}=0.182$ \& Jaccard $\mathrm{R}=0.16$ $\mathrm{p}=0.081$ ) and significant differences between control and migration treatments for non-predators (BrayCurtis $\mathrm{R}=0.478 ; \mathrm{p}=0.0172$ \& Jaccard $\mathrm{R}=0.332, \mathrm{p}=0.0523$ ). The same analysis was conducted using Jaccard distance and is reported in the results section. C1-C5 refer to the control patches while M1-M5 refer to the migration patches.
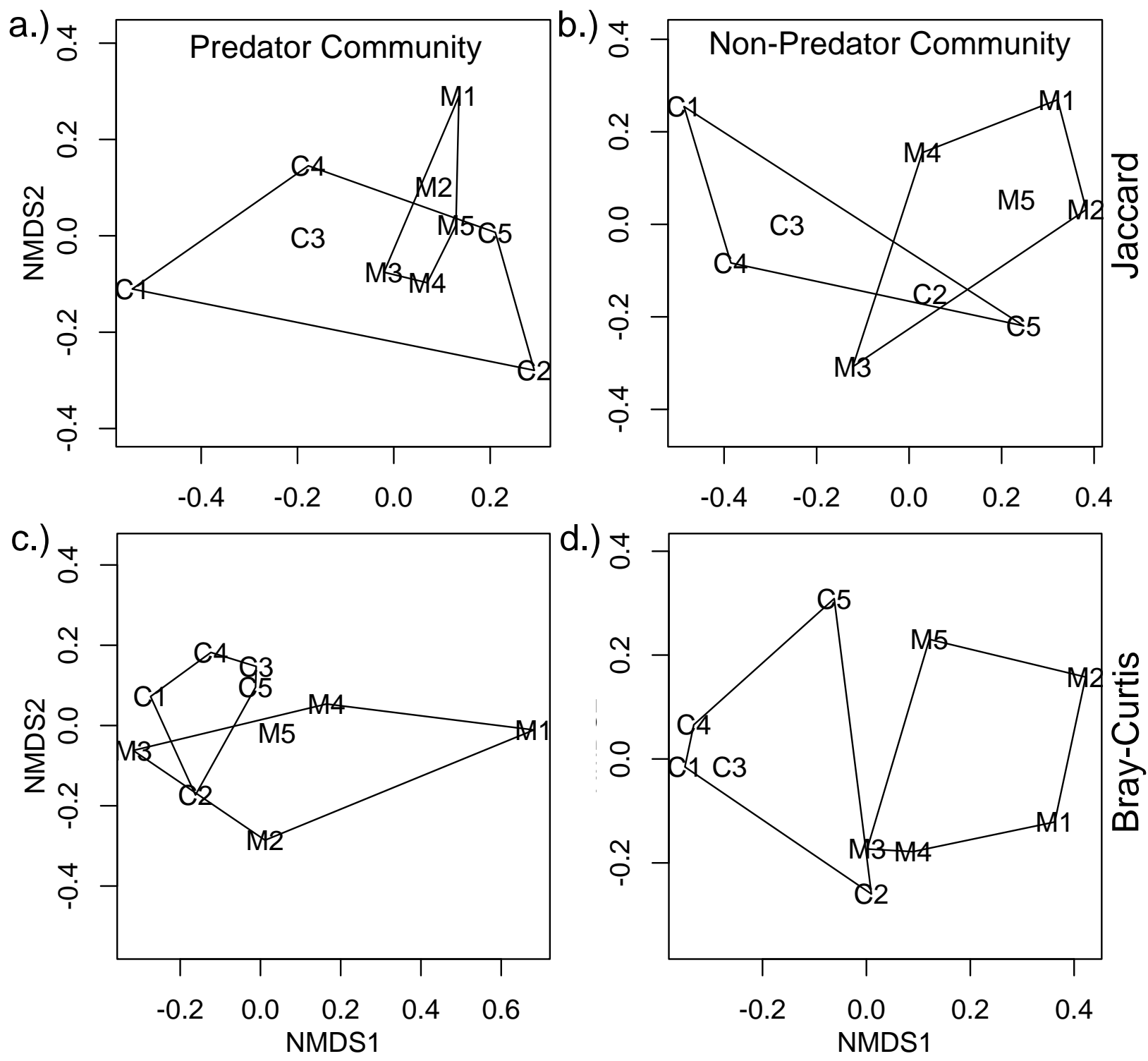

'This article is protected by copyright. All rights reserved.' 
Table Legend

Table 1: Shows the percentage of rare species in the predator and non-predator communities for the control and migration treatment of the experiment. Two definitions of "rare" are used here: first, a morpho-species as a singleton in the dataset (top-half) and second, a given morpho-species as less abundant than the mean abundance of all morpho-species in the community ("relatively rare").

\begin{tabular}{|lc|lc|}
\hline \multicolumn{1}{|c|}{ Predator Community } & $\%$ & Non-Predator Community & $\%$ \\
\hline Singletons & & Singletons & \\
\hline Control & 69 & Control & 49 \\
Migration & 31 & Migration & 51 \\
\hline Relatively Rare & & Relatively Rare & 56 \\
\hline Control & 75 & Control & 44 \\
Migration & 24 & Migration & \\
\hline
\end{tabular}

'This article is protected by copyright. All rights reserved.' 